\title{
PROGRAMME SCIENTIFIQUE
}

\section{Vendredi 4 novembre 2016 SESSION SCIENTIFIQUE 1}

\section{CONCOURS ET PRÉSENTATION DES RÉSIDENTS ET FELLOWS}

Objectifs éducatifs:

A la fin de cette session, les participants seront en mesure de s'assurer d'une actualisation de leurs connaissances sur les projets de recherche récents de la communauté universitaire québécoise en urologie et aussi des projets des jeunes urologues en fellowship.

Modérateurs : Dr Julien Letendre et Dre Katherine Moore

07:55 - 08:10 Mot de bienvenue

Dre Martine Jolivet

Dre Geneviève Nadeau

08:10 - 08:17 The FOXY study: A randomized trial comparing the efficacy and safety of fesoterodine and oxybutynin $\mathrm{XL}$ in children with overactive bladder - Preliminary results

* Sophie Ramsay (fellow), Geneviève Nadeau (urologue), Katherine Moore (urologue), Stéphane Bolduc (urologue)

Division of Urology, CHU de Québec, Université Laval, Québec, QC, Canada

08:17 - 08:24 Outcome comparison of different approaches to selfintermittent catheterization in neurogenic patients: A systematic review

* Samer Shamout (fellow), Xavier Biardeau (fellow), Jacques Corcos (urologue), Lysanne Campeau (urologue)

Jewish General Hospital, Division of Urology, Department of Surgery, McGill University, Montreal, QC, Canada

08:24 - 08:31 Validation de biomarqueur par immunofluorescence : Une approche pour suivre la progression du cancer de la prostate

*Clairefond S (autre), Ouellet V (autre), Péant B (autre), Barrès V (autre), Fragoso G (autre), Mes-Masson AM (autre), Saad F (urologue)

Centre de Recherche du Centre Hospitalier de I'Université de Montréal, Institut du Cancer de Montréal, Montréal, QC, Canada

08:31 - 08:38 $\quad$ Altis $^{\circledR}$ adjustable single-incision sling for female stress urinary incontinence: Mid-term efficacy and satisfaction

*Suzie Adam (autre), Mélanie Aubé (résident), Richard Sioufi (urologue), Le-Mai Tu (urologue)

CHUS Fleurimont, Sherbrooke, QC; Hôpital AnnaLaberge, Châteauguay, QC, Canada

08:38 - 08:45 Assessment of the rate of adherence to international guidelines for androgen-deprivation therapy with external beam radiation therapy: A population-based study

*Hiba Abou-Haidar (résident), Paolo Dell'Oglio (fellow), Sami-Ramzi Leyh-Bannurah (fellow), Zhe Tian (autre), Alessandro Larcher (autre), Giorgio

Gandaglia (urologue), Nicola Fossati (autre), Shahrokh F. Shariat (urologue), Umberto Capitanio (urologue), Alberto Briganti (urologue), Francesco Montorsi (urologue), Markus Graefen (urologue), Fred Saad (urologue), Pierre I. Karakiewicz (urologue) CHUM, Hôpital Saint Luc, Montréal, QC, Canada

08:45 - 08:52

08:52 - 08:59

08:59 - 09:06

09:06 - 09:13

09:13 - 09:20
Effects of chronic lung disease and smoking on prostate cancer mortality after radical prostatectomy *Tal Ben-Zvi (urologue), Moliere Nguile-Makao (fellow), Marc-André Allard (résident), Louis Lacombe (urologue), Yves Fradet (urologue), Vincent Fradet (urologue)

Centre de recherche du CHU de Québec, Université Laval, L'Hôtel-Dieu de Québec, Québec, QC, Canada

Dynamic metabolic imaging of prostate cancer metastasis reveals intra-patient heterogeneous response to systemic therapy

*Fannie Morin (résident), Jean-Mathieu Beauregard (autre), Michelle Bergeron (résident), Molière Nguile Makao (autre), Louis Lacombe (urologue), Vincent Fradet (urologue), Yves Fradet (urologue), Frédéric Pouliot (urologue)

CHU de Québec, Université Laval, Hôtel-Dieu de Québec, Québec, Canada

How to balance the risk of cancer-specific mortality and other-cause mortality in the decision between surgery or observation for patients with T1 kidney cancer

*Vincent Trudeau (résident), Alessandro Larcher (fellow), Paolo Dell'Oglio (fellow), Zhe Tian (autre), Pierre Karakiewicz (urologue)

Cancer Prognostics and Health Outcomes Unit, University of Montreal Health Centre, Montreal, QC, Canada

The use of urodynamics in followup of neurogenic bladders treated with onabotulinum toxin $\mathrm{A}$

*Michelle Bergeron (résident), Katherine Moore (urologue), Geneviève Nadeau (urologue)

CHU de Québec, Institut de réadaptation en déficience physique de Québec, Québec, QC, Canada

Effects of dietary omega-3 fatty acids on tumour growth and immune response in the tramp-C2 prostate tumour model in castrated mice

*Nikunj Gevariya (autre), Alain Bergeron (autre), Marjorie Besançon (autre), Valérie Picard (autre), Karine Robitaille (autre), Yves Fradet (urologue), Vincent Fradet (urologue)

Centre de recherche sur le cancer, Université Laval, Québec, QC, Canada et Laboratoire d'uro-oncologie, Centre de recherche du CHU de Québec, Québec, QC, Canada 
09:20 - 09:27

Épidémiologie, diagnostic, pronostic et prise en charge des tumeurs de vessie chez les patients ayant une vessie neurologique : Revue systématique de la littérature

*Salima Ismail (fellow), Gilles Karsenty (autre), Emmanuel Chartier-Kastler (urologue), Eva Compérat (autre), Morgan Rouprêt (autre), Véronique Phé (autre)

Service d'Urologie, Hôpital Pitié-Salpêtrière, Assistance Publique-Hôpitaux de Paris, Faculté de Médecine Pierre et Marie Curie, Université Paris 6, Paris, France

09:27 - 09:34 La surexpression de IКB-kinase-epsilon (IKK $\varepsilon$ ) favorise la croissance du cancer de la prostate via la phosphorylation de C/EBP- $\beta$ et l'expression du gene de I'IL-6

*Sophie Gilbert (autre), Benjamin Péant (autre), Marc Nicolas Bienz (autre), Cécile LePage (autre), Alexis Poisson (autre), Emilie L'Ecuyer (autre), Nathalie Delvoye (autre), Anne-Marie Mes-Masson (autre), Fred Saad (urologue)

CRCHUM, Institut du cancer de Montréal, Laboratoire du Dr Fred Saad, Montréal, QC, Canada
09:34 - 09:41

Assessment of training on a virtual reality simulator and transfer of skills to the operating theatre: A pilot study

*Mehdi Aloosh (fellow), Yasser A. Noureldin (autre), Sero Andonian (urologue)

McGill University Health Centre, Montreal, QC, Canada

09:41 - 09:48
Associations entre oméga-3 et qualité de vie chez des patients atteints du cancer de la prostate *Moussa Hanane (autre), Nguile-Makao Molière (autre), Allaire Janie (autre), Robitaille Karine (autre), Fradet Vincent (urologue)

CRCHU de Québec, Université Laval, HDQ, Québec, QC, Canada

Pause-santé

Visite des exposants 


\section{PROGRAMME SCIENTIFIQUE}

\section{SESSION SCIENTIFIQUE 5}

\section{CONCOURS ET PRÉSENTATION DES RÉSIDENTS ET FELLOWS}

Objectifs éducatifs

A la fin de cette session, les participants seront en mesure de s'assurer d'une actualisation de leurs connaissances sur les projets de recherche récents de la communauté universitaire québécoise en urologie et aussi des projets des jeunes urologues en fellowship.

Modérateurs : Dr Yves Caumartin et Dre Annie Lessard

13:45 - 13:52

13:52 - 13:59

13:59 - 14:06
The risk of urinary retention following robot-assisted radical prostatectomy and its impact on continence outcomes

*Mansour Alnazari (fellow), Marc Zanaty (fellow), Assaad El-Hakim (urologue), Kevin C. Zorn (urologue) Hôpital Saint-Luc, Hôpital Sacré Coeur de Montréal, Montréal, QC, Canada

Value of voiding cysto-urethrogram in assessment of high-grade post-natal hydronephrosis

*Amr Hodhod (fellow), J.P. Capolicchio (urologue), Roman Jednak (urologue), Mohamed El-Sherbiny (urologue)

Department of Pediatric Surgery, Urology, Montreal Children`s Hospital, McGill University, Montreal, QC, Canada

Cytokine/FER-activated androgen receptor (PY223AR): A predictive biomarker of prostate cancer progression

*Turki Altaylouni ${ }^{1}$ (autre), Fatima Zahra Zouanat (autre), Eleonora Scarlata ${ }^{1}$ (autre), Lucie Hamel (autre), Fadi Brimo' (autre), Louis Bégin ${ }^{2}$ (autre), Armen Aprikian ${ }^{1}$ (urologue), Simone Chevalier ${ }^{1}$ (autre) 'Urologic-Oncology Research Team, Research Institute of McGill University Health Centre, Montreal, QC, Canada; ${ }^{2}$ Sacré Coeur Hospital, Montreal, QC, Canada
14:06 - 14:13

$14: 13-14: 20$

$14: 20-14: 27$

$14: 27-14: 34$

$14: 34-14: 41$

$14: 41-15: 35$
Effect of diabetes and metformin on recurrence and progression in patients with non-muscle-invasive bladder cancer

* Samer Traboulsi (urologue), Bassel Bachir (urologue), Faysal Yafi (urologue), Franck Bladou (urologue), Simon Tanguay (urologue), Armen Aprikian (urologue), Wassim Kassouf (urologue)

McGill University Health Centre, Montreal, QC, Canada

Cytotoxic T lymphocyte CD8+, CD3+, and immunoscore as prognostic markers in patients after radical cystectomy

*Alice Yu (résident), Jose Joao Mansure (autre), Shraddha Solanki (autre), Fadi Brimo (autre), Wassim Kassouf (urologue)

Division of Urology, Department of Surgery, McGill University, Montreal, QC, Canada

Factors associated with the management of metastatic castration-resistant prostate cancer in a real-life setting in Quebec

*Halima Lahcene (résident), Armen Aprikian (urologue), Marie Vanhuyse (autre), Franck Bladou (urologue), Noémie Prévost (autre), Jason Hu (autre), Fabio Cury (autre), Wassim Kassouf (urologue), Sylvie Perreault (autre), Alice Dragomir (autre)

Hôpital Général de Montréal (HGM), Hôpital Général Juif (HGJ), Montréal, QC, Canada

Assessment of battery life of the second-generation implantable pulse generator in a practice of highvolume implanters

*Daniel Liberman (urologue), Marta Johnson-Mitchell (autre), Steven W. Siegel (autre)

Metro Urology, Woodbury, MN, United States

Prognostic factors of chronic kidney disease in patients with posterior urethral valves

*Monica Salgado (autre), Yann Chaussy (urologue), Anne-Marie Houle (urologue), Pierre-Alain Hueber (résident), Diego Barrieras (urologue), Julie FrancGuimond (urologue)

CHU Sainte-Justine, Montreal, QC, Canada

Pause-santé

Salon des exposants 


\section{PROGRAMME SCIENTIFIQUE}

\section{SESSION SCIENTIFIQUE 6}

\section{CONCOURS ET PRÉSENTATION DES RÉSIDENTS ET FELLOWS}

Objectifs éducatifs

A la fin de cette session, les participants seront en mesure de s'assurer d'une actualisation de leurs connaissances sur les projets de recherche récents de la communauté universitaire québécoise en urologie et aussi des projets des jeunes urologues en fellowship.

Modérateurs : $\quad$ Dr Samer Hanna et Dr Frédéric Soucy

15:35 - 15:41 Prognostic factors in radical prostatectomy and permanent seed brachytherapy for low- and intermediate-risk prostate cancer: A comparative study

*Guila Delouya (autre), Daniel Taussky (autre), Véronique Ouellet (autre), Fred Saad (urologue)

Centre hospitalier et Centre de Recherche de I'Université de Montréal (CHUM-CRCHUM), Montréal, QC, Canada

15:41 - 15:48

Urothelial cells express a functional succinate receptor GPR91

Monica Velasquez (autre), Philippe Cammisotto (autre), *Lysanne Campeau (urologue)

Lady Davis Institute for Medical Research, Division of Urology, Department of Surgery, McGill University, Montreal, QC, Canada

15:48 - 15:55 Safety and durability of sacral neuromodulation in the geriatric population: A sub-analysis from the INSITE trial

*Daniel Liberman' (urologue), Tomas L. Griebling ${ }^{2}$ (autre), Steven W. Siegel ${ }^{1}$ (autre)

${ }^{1}$ Metro Urology, Woodbury, MN, United States; ${ }^{2}$ University of Kansas School of Medicine, Kansas City, KS, United States
15:55 - 16:02

16:02 - 16:09

16:09 - 16:16

$16: 16-16: 23$

\section{metabolic syndrome}

Succinate in voiding dysfunction associated with

*Monica Velasquez Flores (autre), Philippe Cammisotto (autre), Lysanne Campeau (urologue)

Lady Davis Institute for Medical Research, Montreal, QC, Canada 


\section{PROGRAMME SCIEN'TIFIQUE}

\section{Samedi 5 novembre 2016 SESSION SCIENTIFIQUE 9}

Objectifs éducatifs

A la fin de cette session, les participants seront en mesure de bénéficier $\mathrm{d}^{\prime}$ une mise à jour sur les projets de recherche récents de la communauté universitaire québécoise en urologie et des projets de jeunes urologues en fellowship.

\section{Modérateurs : \\ Dr Serge Carrier et Dr Hugues Widmer}

08:15 - 08:22

Résultats initiaux de la cystectomie avec dérivation urinaire intracorporelle robot-assistée pour cancer *Frank Bladou (urologue), Marcangione (autre), Cerentola (fellow), Ploussard (fellow)

Hopital Général Juif, Université McGill, Montréal, QC, Canada

08:22 - 08:29

08:29 - 08:36

\begin{abstract}
Changes in the levels of prostate-specific antigen and testosterone profile over time in a cohort of patients treated with active surveillance for prostate cancer *Ahmed S. Zakaria (fellow), Alice Dragomir (autre), Noémie Prévost (autre), Wassim Kassouf (urologue), Simon Tanguay (urologue), Armen G. Aprikian (urologue) Division of Urology, Cancer Centre, Montreal University Health Centre, Montreal, QC, Canada
\end{abstract}

Anticiper la réponse thérapeutique des patients atteints d'un cancer de la prostate: Développement d'outils micro-fluidiques pour une médicine personalisée *Benjamin Péant (autre), Abdul Lateef (fellow), Kayla Simeone (autre), Euridice Carmona (autre), Jennifer Kendall-Dupont (autre), Adriana Mari Orimoto (autre), Thomas Gervais (autre), Anne-Marie Mes-Masson (autre), Fred Saad (urologue)
08:36 - 08:43

08:43 - 08:50

08:50 - 08:57

08:57 - 09:04
Développement d'une technique de microscopie à bioluminescence pour évaluer, ex-vivo et cellule par cellule, l'héterogeneite de la réponse tumorale aux anti-androgenes

*Frédéric Pouliot (urologue), Pallavi Jain (autre), Bertrand Neveu (autre), Yves Fradet (urologue)

Université Laval et CHU de Québec, Québec, QC, Canada

Large, single-centre experience with postoperative adjuvant or salvage radiotherapy for prostate cancer *Daniel Taussky (autre), Guila Delouya (autre), Carole Lambert (autre), Maroie Barkati (autre), Marie-Claude Beauchemin (autre), Jean-Paul-Bahary (autre), Cynthia Ménard (autre), Kevin C. Zorn (urologue), Fred Saad (urologue)

Centre hospitalier de I'Université de Montréal (CHUM), Montréal, QC, Canada

Video presentation: Posterior urethroplasty with a gracilis muscle flap

*Daniel Liberman (urologue), Sean P. Elliott (autre) University of Minnesota, Minneapolis, MN, United States

Video presentation: Re-establishing vaginal apical support after vaginal hysterectomy using the modified Mayo-McCall culdoplasty

*Daniel Liberman (urologue), John B. Gebhart (autre) Mayo Clinic, Rochester, MN, United States 